\title{
EDUCACIÓN AMBIENTAL POPULAR PARA EL MANEJO SUSTENTABLE DE RECURSOS NATURALES EN UNA LOCALIDAD RURAL DEL SUBTRÓPICO MEXICANO
}

\section{Popular environmental education for natural resources sustainable management at a rural community in the Mexican Subtropics}

Etztli Itzel Morales Reyes etizel114@hotmail.com

Universidad Autónoma del Estado de México, Toluca, Estado de México, México

Jesús Gastón Gutiérrez Cedillo

jggc1321@yahoo.com.mx

Universidad Autónoma del Estado de México, Toluca, Estado de México, México

Xanat Antonio Nemiga

xanynemiga@hotmail.com

Universidad Autónoma del Estado de México, Toluca, Estado de México, México

Miguel Ángel Balderas Plata mplata@colpos.mx

Universidad Autónoma del Estado de México, Toluca, Estado de México, México

Artigo recebido em 28/05/2014 e aceito para publicação em 03/12/2015

RESUMEN: El objetivo principal de este trabajo fue diseñar, ejecutar y evaluar un programa de educación ambiental popular para el manejo sustentable de recursos naturales, a fin de mejorar relaciones entre la comunidad y su entorno natural, a través de la acción participativa, en una localidad rural campesina ubicada en una zona de ecotono, al suroeste del Estado de México. Este trabajo buscó promover una visión crítica, en el conocimiento de los problemas del medio natural y social en su conjunto. Se establecieron las siguientes etapas metodológicas: 1)Investigación, se identificaron las necesidades de aprendizaje y los problemas de interés de la comunidad; 2)Educación, se realizó una propuesta curricular de educación ambiental por medio de dos programas impartidos en doce talleres; 3)Acción, se desarrollaron experiencias de educación ambiental popular, mediante talleres con tres grupos: niños, mujeres y hombres; 4)Acción -Reflexión- Acción, se evaluaron los resultados de las experiencias sobre educación ambiental. En los resultados de la evaluación final de los talleres, los participantes fueron capaces de definir, ejemplificar y plantear alternativas; además, realizaron acciones para el manejo sustentable de recursos naturales en su comunidad.

Palabras Clave: Educación Ambiental Popular; Manejo Sustentable; Recursos Naturales; Localidad Rural; Subtrópico Mexicano.

ABSTRACT: The main objective of this study was to design, implement and evaluate a popular environmental education program for natural sustainable resource management, directed to improve relations between community and their natural environment, through participatory action, at a rural community located in ecotone zone,

DOI: http://dx.doi.org/10.1590/1982-451320160103 
southwest of the State of Mexico. This work was searching to promote a critical vision, on knowledge of natural and social environmental problems as a whole. The following methodological steps were established: 1) Research, in which will be indentified the learning needs of the community, and issues of interest to the community, 2) Education, in which a curriculum proposal for environmental education was conducted, through two programs offered means by workshops, 3) Action, popular environmental education experiences were developed through twelve workshops with three groups: children, women and men, 4) Action-Reflection-Action, in which the results of environmental education experiences were evaluated. As a result, it was observed that the participants were able to define, model and propose alternatives for natural sustainable resource management at their community.

Keywords: Popular Environmental Education; Sustainable Management; Natural Resources; Rural Community; Mexican Subtropics.

\section{INTRODUCCIÓN}

La agricultura comercial genera alto impacto ambiental que se manifiesta en problemas de erosión, compactación, salinización, acidificación, sedimentación, contaminación de suelos, agua, atmósfera, pérdida de biodiversidad, deforestación, desertificación y efecto invernadero, con graves efectos en la salud humana (JUAN, 2007).

Progreso Hidalgo es una comunidad rural del subtrópico mexicano donde realizan actividades a que combinan agricultura comercial y la agricultura familiar, (JUAN, 2007). En estudios realizados por Juan (2003, 2006, 2007 y 2009), se argumenta que en la región agrícola del sureste del Estado de México, en la que se encuentra la zona de estudio del presente trabajo, el manejo del ambiente es importante para mitigar los efectos de los riesgos desde el punto de vista económico y ecológico; ya que cuando las familias de la región entran al sistema de producción capitalista, se enfrentan a condiciones que de manera directa e indirecta influyen en el bienestar de toda la comunidad.

Así mismo Juan et al., 2009 caracterizaron y diagnosticaron los sistema de barrancos del río Calderón que forman parte del área de estudio de este trabajo, con el fin de conocer el potencial natural y sociocultural que estos sistemas ofrecen; y de estudiar los mecanismos utilizados por los pobladores para el manejo de los recursos naturales existentes, esto con la finalidad de proponer acciones que propicien el turismo alternativo como estrategia para el desarrollo local y regional, coadyuvando simultáneamente en la conservación de la estructura y funcionamiento del sistema.

La participación de los pobladores es importante para identificar diversas problemáticas ambientales que existen en la zona de estudio, así como para presentar alternativas a los problemas planteados y emprender propuestas para modificar la relación del ser humano con el ambiente a nivel local.

La educación ambiental popular para el manejo sustentable de recursos naturales, se propuso como una alternativa con visión pedagógica y acción participativa, que aspiró a que los habitantes de la comunidad de Progreso Hidalgo, adquieran una nueva actitud con la que modifiquen las relaciones que históricamente han causado daño a su ambiente, y así propiciar que los habitantes de la comunidad se inserten en un sistema de valores y un esquema social de relaciones más solidarias, cooperativas, autónomas y equitativas.

La investigación agrícola que históricamente se ha realizado en México se ha enfocado casi exclusivamente hacia la productividad de los recursos naturales (suelo, agua, clima y germoplasma) de las regiones más productivas, la que se clasifica como revolución verde o agricultura comercial. Sin embargo, en esas regiones se ha ignorado la investigación para el conocimiento de esos recursos per se; también se ha descuidado el estudio de los efectos negativos de las prácticas agrícolas: es decir, de las externalidades ecológicas (TURRENT; CORTÉS, 2005).

Por tanto, parece sensato abrazar como país, el paradigma de la agricultura alternativa, ya que esta representa una opción de desarrollo sustentable en el campo mexicano. 
La Comisión Mundial del Medio Ambiente y el Desarrollo define al desarrollo sustentable como: "Aquel desarrollo que satisface las necesidades presentes sin comprometer la capacidad de que las futuras generaciones puedan satisfacer las propias", esto implica poner en marcha un proceso de crecimiento económico, con equidad social y conservación de la integridad de los ecosistemas y del ambiente en su conjunto (CÁRDENAS, 2009).

La educación popular es una corriente educativa que se caracteriza por ser, a la vez un fenómeno sociocultural y una concepción de educación que tiende a la sustentabilidad.

Este sistema educativo constituye una propuesta pedagógica importante como fundamento para esta investigación, ya que la bibliografía generada en torno a ella, es considerada como una corriente educativa, un campo teórico-práctico coherente que puede incluir diversas modalidades posibles y abarcar múltiples niveles de prácticas educativas que pueden ser: comunitarias, grupales, formales, no formales, con personas adultas, con niños, niñas y jóvenes (SAUVÉ, 2006).

La propuesta metodológica y pedagógica de la Educación Ambiental Popular retoma presupuestos como son: la reflexión de lo particular a lo general, partir de la práctica a la teoría y después renovarla, la valorización y revalorización del saber popular, una asunción amplia del proceso educativo, con la participación de sectores cada vez más amplios de la sociedad. Además de los referentes metodológicos señalados, la Investigación Acción Participativa y la Evaluación Rural Participativa, constituyen presupuestos metodológicos importantes para el desarrollo de experiencias vinculadas al desarrollo rural sustentable, las que convergen en cuanto al papel protagónico y participativo de la población local, la comunidad o el espacio rural, como escenario para realizar este tipo de experiencias (REYES, 1994).

La utilización de Investigación Participativa (IP) como herramienta facilita el proceso de evaluación, dentro de esta es importante destacar cinco elementos: el carácter educativo de la IP, el papel de esta en el proceso de transformación y producción de conocimientos, la IP como método dialógico, el papel del conocimiento local y al componente participativo dentro de la IP. (FALS; RODRÍGUEZ 1987).

\section{OBJETIVOS}

El objetivo general de este trabajo fue implementar y evaluar la inclusión de un programa de educación ambiental popular, para el manejo sustentable de recursos naturales en Progreso Hidalgo, mediante talleres en grupos.

Los objetivos específicos son: 1) Caracterizar la zona de estudio e identificar las necesidades de los habitantes locales sobre educación ambiental; 2) Diseñar una propuesta, encaminadaa la introducción del "Modelo Pedagógico de Educación Ambiental Popular para el Manejo Sustentable de Recursos Naturales"; 3) Desarrollar experiencias comunitarias en Progreso Hidalgo, seleccionando los casos de estudio correspondientes, e implementándolos, monitoreándolos y extrayendo las experiencias adecuadas; 4) Evaluar los resultados de las experiencias sobre educación ambiental popular establecidas y monitoreadas.

\section{ÁREA DE ESTUDIO}

Desde el punto de vista fisiográfico, en el contexto espacial del territorio del Estado de México, esta región se encuentra en una zona de ecotono y presenta características específicas en sus componentes físicos y biológicos, con respecto a otras regiones del territorio estatal.

Progreso Hidalgo está situado en una región fresera, ubicada entre la Provincia de las Serranías Meridionales y la Provincia de la Depresión del Río Balsas, perteneciente a la Región Caribeña del Reino Neotropical y caracterizada por la presencia de climas cálidos y semicálidos (GOBIERNO DEL ESTADO DE MÉXICO, 1995 Y 2000). Cuenta con una morfología accidentada, donde la topografía y las condiciones del relieve han originado variadas estructuras, como los sistemas de barrancos, lomeríos y mesetas. Esta región, forma parte de la cuenca del Río Grande de Amacuzac, que nace en las pendientes del Volcán Xinantécatl dentro de la Región Hidrológica del Río Balsas. 
El clima corresponde al grupo $\mathrm{A}(\mathrm{C}) \mathrm{wg}$, que de acuerdo con el sistema de clasificación climática de Koppen, modificado por García, corresponde a un clima tropical lluvioso, semicálido, (de transición entre el clima cálido y templado) con elevadas temperaturas durante la primavera y el verano. El suelo predominante es Vertisol Pélico; desde el punto de vista agrícola, son fértiles y tienen capacidad agrológica para usarse en el manejo de una alta diversidad de cultivos como es el caso de la fresa, cultivo introducido en 1972 (INEGI, 1982).

La vegetación natural es característica del bosque tropical caducifolio, las especies más representativas son tepehuaje, amate, palo blanco, cazahuate, guaje, huizache y mezquite, árboles que se encuentran en las zonas abruptas y en las barrancas. Los animales representativos corresponden a la fauna del bosque tropical caducifolio, y representan una componente importante en la dieta de muchas familias (JUAN, 2006).

Cuando se estableció la localidad de Progreso Hidalgo, debido a sus características físicas se iniciaron diversos cambios en el ambiente, principalmente aquellos derivados de la preparación de tierras y campos de cultivo. En términos de los propios pobladores de la localidad, ellos afirman que el sitio originalmente "era una espinera"; que en 1948 cambió para dar paso a la agricultura de temporal y empezar a cultivar fríjol y maíz, destinados principalmente a alimentar a las familias campesinas (JUAN, 2006). Con la expansión de parcelas de cultivo hacia las zonas forestales, se incrementó el corte de árboles con el fin de permitir la entrada de luz y otros efectos derivados del sistema de cultivo conocido como roza, tumba y quema; basado en el conocimiento refinado que tienen los pobladores acerca de su entorno (JUAN, 2006).

En 1950 se conduce agua a la localidad, mediante un sistema de abastecimiento, almacenamiento, conducción y distribución por medio de zanjas, canales o "apancles" que conducen agua hacia los terrenos de cultivo; sistema de riego que aún prevalece y que es pilar fundamental para la economía de las familias campesinas (GOBIERNO DEL ESTADO DE MÉXICO, 1958). En los cuerpos de aguas permanentes, estacionales y terrenos anegables, se construyeron sistemas y represas para el almacenamiento, conducción y distribución de agua, que coadyuvan en el manejo del recurso hídrico y son importantes para el riego de cultivos comerciales en terrenos agrícolas de propiedad privada y ejidal, favoreciendo la agrobiodiversidad en la región. En la localidad existen 12 cuerpos de agua permanentes con diferentes capacidades y variados usos, dos estanques para la producción acuícola y 12 terrenos sujetos a inundación.

A partir de la introducción del sistema de riego los pobladores empezaron a combinar y establecer nuevos cultivos como el tomate, pepino, gladiolo, camote, cebolla, cacahuate, calabaza, frijol de riego, chilacayote y chayote; esto con dos finalidades, la primera abastecer de alimento a las familias, y la segunda comercializar los productos agrícolas en la región. En la década de 1970 se introdujo el cultivo de la fresa, el que a partir de esa fecha se empezó a intercalar con otros cultivos, para 1990 se empieza a sembrar el cempasúchil y del 2000 hasta la actualidad la flor conocida como aster. Para 2004 y 2005 el manejo del ambiente incluidos barrancas, cuerpos de agua, suelo y el establecimiento de nuevos cultivos, trajo como consecuencia que el $95 \%$ de los pobladores se dediquen a cultivar y comercializar fresa y gladiola. Los pobladores tratan así de obtener mejores resultados y ganancias, por lo que cada periodo agrícola implementan y utilizan nuevos insumos agroquímicos, lo que lleva a la comunidad hacia un sistema de cultivo industrial, de acuerdo a una tendencia global.

En Progreso Hidalgo la tenencia de la tierra es ejidal, actualmente existe una población total de 811 habitante, de los cuales 406 son masculinos y 405 femeninas, los ciudadanos se dividen en 408 menores de edad y 403 adultos, de ellos 44 tienen más de 60 años, la media de escolaridad entre la población es de secundaria( INEGI, 2010). En cuanto a los servicios cuentan con una escuela primaria, luz y agua potable (INEGI, 2010).

La economía en la región es campesina y el modo de subsistencia es agrícola. Shanin (1976) afirma que la división del trabajo se vincula con la estructura familiar, considerando la edad y el sexo. Los campesinos de la localidad pueden subsistir debido a 
que a lo largo del año se adaptan a las condiciones del ambiente y del mercado mediante formas de cohesión social y organización que derivan de la familia y sus relaciones entre sí (JUAN, 2006). La organización y división del trabajo, de acuerdo con la edad y el género dentro del ámbito familiar, favorecen una amplia diversidad de actividades agrícolas y domésticas. Asimismo mantiene constante la fuerza de trabajo asalariada y la ayuda mutua dentro de la región. Cada integrante de la familia realiza diferentes actividades durante los ciclos agrícolas.

\section{MATERIALES Y MÉTODOS}

La investigación se basó en la Educación Ambiental Popular para el Manejo Sustentable de Recursos Naturales en Progreso Hidalgo, y se fundamentó enlametodologíadeInvestigaciónAcción Participativa, por lo que incluyó la participación social de los habitantes de Progreso Hidalgo, y se basó en la recuperación de las inquietudes expresadas en doce talleres comunitarios.

Figura 01 - Etapas del proceso metodológico de la investigación

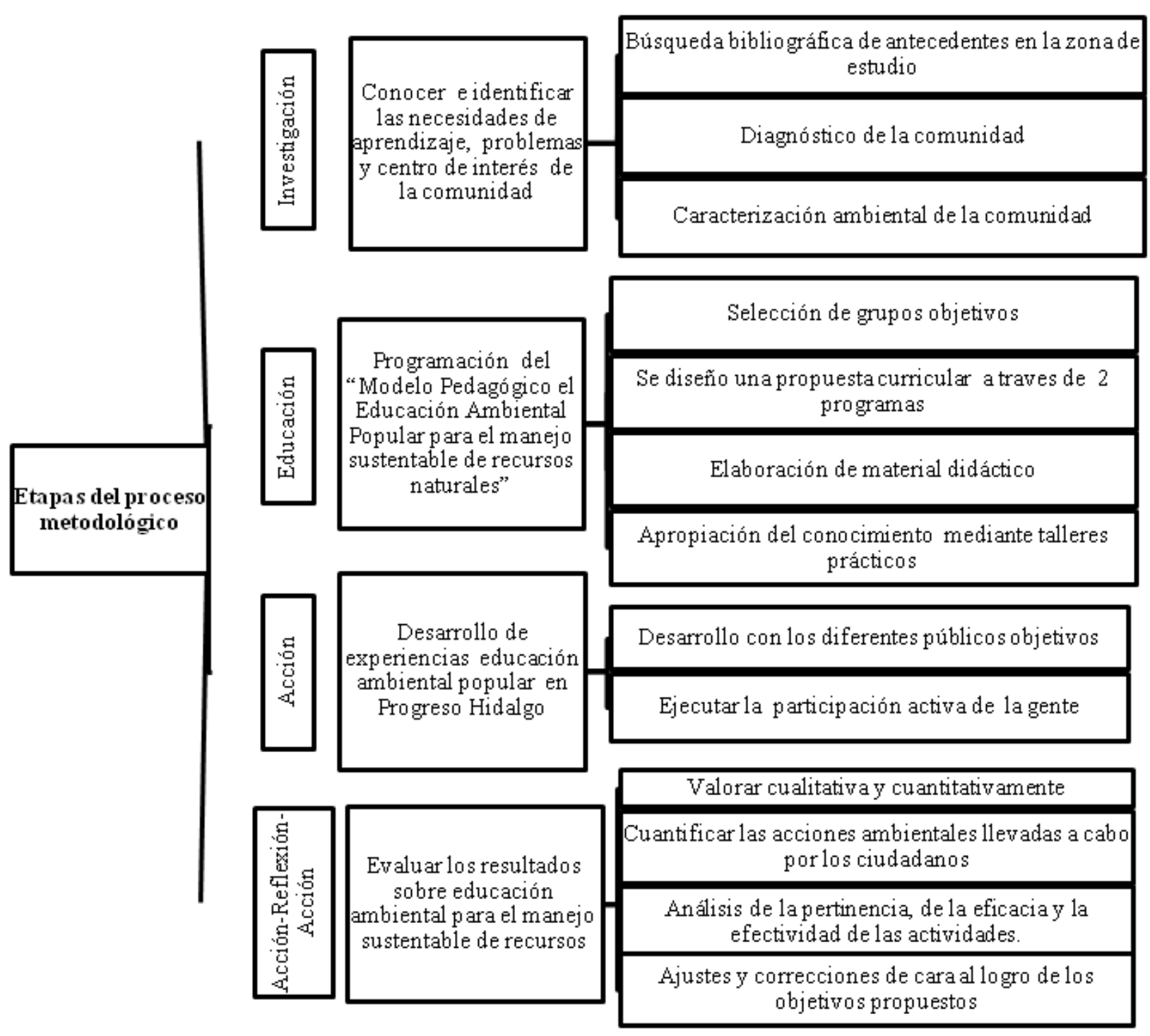

Fuente: Elaboración propia, 2014. 


\section{RESULTADOS Y DISCUSIÓN}

Los resultados se presentan de acuerdo a las etapas metodológicas de la investigación.

\section{Fase de Investigación}

En esta fase se realizó la búsqueda bibliográfica y recorridos sistémicos de observación directa en campo, con la finalidad de elaborar, la caracterización de la comunidad, y se constató que debido a que esta localidad se encuentra ubicado en una zona de ecotono, existe una gran diversidad de recursos naturales; con un clima propicio para el desarrollo de múltiples cultivos, lo que favorece su desarrollo agrícola, como actividad económica importante.

En las actividades agrícolas se utilizan grandes cantidades de agroquímicos, que de manera directa e indirecta inciden en la calidad del ambiente de esta comunidad. La vegetación natural de la región ha sido afectada por quemas no controladas, tala selectiva de algunas especies y por el hecho de que algunos ejidatarios tratan de ampliar los límites de sus parcelas, abriendo espacios naturales como nuevas áreas para el pastoreo y para el cultivo de especies agrícolas.

En esta etapa de la investigación se elaboró un análisis de Fortalezas, Oportunidades, Debilidades y Amenazas (FODA) dirigido a determinar los conocimientos, intereses y necesidades de los habitantes locales, en relación con sus problemas ambientales a través de un proceso participativo.

Con el análisis FODA se establecieron las estrategias FO, FA, DO, DA las cuales ayudaron a proponer mejoras en el área de estudio. La estrategia FO para maximizar las fortalezas y las oportunidades en la comunidad, fue útil para implementar programas de aprovechamiento sustentable de los recursos naturales. Con la estrategia FA para minimizar las amenazas y maximizar las fortalezas, se estableció un vínculo entre las cualidades geográficas y ambientales con las que cuenta la comunidad y el manejo inadecuado de prácticas agrícolas, a fin de establecer un equilibro, para mejorar las condiciones ambientales, y establecer alternativas para la actividad agrícola. En la estrategia DO que se diseño para minimizar las debilidades y maximizar las oportunidades, se resaltó el establecimiento de programas de educación y desarrollo local para la conservación el ambiente y la calidad de vida de la población. En la estrategia DA para minimizar las debilidades y las amenazas se incluyó la elaboración de diagnósticos mediante acción participativa de la comunidad, con el fin de lograr mayor organización interna, y generar manejo sustentable de los recursos.

Este análisis formó parte del diagnóstico sobre la situación actual real de los habitantes de Progreso Hidalgo; a partir de un ejercicio reflexivo, se identificaron límites espaciales, recursos naturales disponibles, algunos conflictos socioambientales, valores habituales y estructura de la población, entre otros. Con base en esta experiencia se desglosaron formas y motivos de actuación, y se propusieron acciones dentro de la definición del plan de actividades. Esta etapa fue fundamental, ya que como señala Balcázar, (2003) este tipo de investigación trata de promover la participación de los miembros de comunidades, en la búsqueda de soluciones a sus propios problemas, para contribuir a incrementar el grado de control que ellos tienen sobre aspectos relevantes en sus vidas (incremento de poder o empoderamiento).

Esto coincide con lo señalado por Selener, (1997) quien conceptualiza la Metodología de Investigación Acción Participativa como "un proceso por el cual, miembros de un grupo o una comunidad oprimida, colectan y analizan información; y actúan sobre sus problemas con el propósito de encontrar soluciones y promover transformaciones políticas y sociales"

\section{Fase de Educación}

Esta etapa se fundamentó en el diagnóstico, previa caracterización de la comunidad; a través de éste se determinaron las actividades a realizar, y los grupos objetivo a los que podría dirigirse; ya que como señalan Chávez; Daza (2003), el ámbito de aplicación es un punto indispensable al diseñar una investigación que requiere utilizar la IAP, el cual 
corresponde al espacio físico y social donde se llevan a cabo las maniobras del proyectos y elementos que influyen al momento de desarrollar las actividades.

Los criterios que se tomaron en cuenta para la realización de los talleres y la conformación de los grupos fueron principalmente la organización social y agrícola, las que derivan de la familia. Es por esto que se determinaron tres grupos que incluyeron a integrantes de los diversos grupos de edad y género: niños, mujeres y hombres. Se decidió iniciar los talleres con los niños, ya que en las pláticas sostenidas con los pobladores, ellos manifestaron que era mejor iniciar con niños, dado que algunos adultos se mostraban escépticos de lo que se podría lograr con los talleres. Al iniciar con los niños, ellos podrían despertar el interés a otros miembros de la familia.

Esta estrategia funcionó, ya que después de empezar a impartir los talleres, las madres de los niños tuvieron inquietud por saber más acerca de los temas ambientales y decidieron formar un grupo con el que comenzamos a impartir los temas acerca de los recursos del agua, suelo y vegetación. Después de impartir los talleres con el grupo de niños y mujeres se logró formar un grupo con los señores adultos de la comunidad; este grupo fue el más incrédulo y al principio se mostraba desconfiado, sólo poco a poco decidieron integrarse.

En cuanto a las temáticas sociales que se abordaron en los talleres, estas fueron propuestas por los integrantes de los grupos después de la realización de un análisis de fortalezas y debilidades. Esta técnica participativa se considera un factor fundamental para la fase de educación. Como afirman Chávez; Daza (2003), en el adecuado funcionamiento de los grupos radica el éxito de la investigación, lo que en términos descriptivos comprende cualquier clase de asociación en la que sus integrantes se reúnen, con el fin de llevar a cabo una acción para mejorar sus condiciones.

Los nombres de los talleres fueron designados posteriormente, ya que básicamente abarcaron los mismos tópicos para los diversos grupos, solo se dio énfasis en lo que cada grupo propuso: a los niños les interesaban las relaciones humanas; a las mujeres la comunicación para aumentar la comprensión; y a los hombres la organización comunitaria para el desarrollo local. Chávez; Daza (2003) señalan que la dinámica grupal incluye la subjetividad de cada uno de sus componentes, y las variables que aparecen en la vida de estos conjuntos. Al darse la interacción entre sus miembros, se genera una multiplicidad de intersubjetividades, que amplía el análisis sobre los temas investigados.

En esta fase se construyó un Programa de Educación Ambiental, de acuerdo con Chávez; Daza (2003), es importante la utilización de técnicas para recopilar, sistematizar, divulgar y retroalimentar la información (escrita, visual y oral); sobre lugares, procesos y situaciones que se investigan.

En este proceso se optó por la realización de talleres, que como indica Gisho (1999), el taller es reconocido como un instrumento válido para la socialización, la transferencia, la apropiación y el desarrollo de conocimientos, actitudes y competencias, de una manera participativay pertinente a las necesidades y cultura de los participantes.

En los talleres se abordaron temas relacionados con aspectos de la comunidad que los pobladores consideran que han sido más afectados por la agricultura comercial; y que son sumamente importantes para las actividades agrícolas. Estos aspectos de interés fueron: el suelo, el agua y la vegetación. Se elaboró el material didáctico para los talleres y se aplicó un cuestionario para evaluar la percepción ambiental de los participantes.

\section{FASE DE ACCIÓN}

En esta etapa se realizó la puesta en marcha del Programa de Educación Ambiental Popular para el Manejo Sustentable de Recursos Naturales, por medio de talleres prácticos en los que se abordaron los temas que se observan en la Tabla 01 . 
Educación Ambiental Popular Para El Manejo Sustentable De Recursos Naturales En Una Localidad Rural Del Subtrópico Mexicano Etztli Itzel Morales Reyes, Jesús Gastón Gutiérrez Cedillo, Xanat Antonio Nemiga, Miguel Ángel Balderas Plata

Tabla 01 - Temas de los talleres del Programa de Educación Ambiental Popular

\begin{tabular}{|l|l|l|l|}
\hline $\begin{array}{l}\text { Talleres Ambientales } \\
(9 \text { talleres })\end{array}$ & Grupos de Trabajo & $\begin{array}{l}\text { Talleres sobre Temas } \\
\text { Sociales (3 talleres })\end{array}$ & Grupos de trabajo \\
\hline Recursos del suelo & $\begin{array}{l}\text { Grupo de niños } \\
\text { Grupo de mujeres } \\
\text { Grupo de hombres }\end{array}$ & Relaciones humanas & Grupo de niños \\
\hline Recursos del agua & $\begin{array}{l}\text { Grupo de niños } \\
\text { Grupo de mujeres } \\
\text { Grupo de hombres }\end{array}$ & Comunicación & Grupo de mujeres \\
\hline Recursos de la vegetación & $\begin{array}{l}\text { Grupo de niños } \\
\text { Grupo de mujeres } \\
\text { Grupo de hombres }\end{array}$ & $\begin{array}{l}\text { O r g a n i z a c i ó n } \\
\text { comunitaria y solución } \\
\text { de conflictos }\end{array}$ & Grupo de hombres \\
\hline
\end{tabular}

Fuente: Elaboración propia.

En esta fase de la investigación los participantes aprendieron a desarrollar una conciencia crítica que les permite identificar las causas de sus problemas y sus posibles soluciones. Como afirma Balcázar (2003) el propósito es enseñar a la gente a descubrir su propio potencial para actuar, liberándoles de estados de dependencia y pasividad previos, y llevarlos a comprender que la solución, está en el esfuerzo que ellos mismos puedan aportar para cambiar el estado de cosas.

\section{Fase de Acción-Reflexión-Acción}

En esta fase del estudio se examinaron los resultados de las experiencias sobre educación ambiental popular para el manejo sustentable de recursos naturales. Primero se evaluó la percepción ambiental de los participantes, antes y después de los talleres, utilizando un cuestionario, que abordo los siguientes aspectos:

a. Conocimiento acerca de la visión ambiental y de problemas ambientales.

b. Reconocimiento de la extensión con que se producen los problemas ambientales

c. Visión que tienen los sujetos sobre el medio ambiente y los problemas que en él se presentan (PADILLA; LUNA, 2003).

d. Actitud ambiental y decisión a incorporarse al cambio (PADILLA; LUNA, 2003).

A continuación se presentan los resultados de este cuestionario. Primeramente la visión del medio ambiente y la percepción que poseen los individuos sobre la definición de medio ambiente $\mathrm{y}$ sus dimensiones. Esta es importante ya que representa la capacidad para percibir adecuadamente los diferentes elementos que conforman el medio en el que todos nos desenvolvemos. Esta visión condiciona las actitudes y sensibilidades; e influyen considerablemente en la orientación y regulación de las acciones humanas hacia el entorno.

Los resultados obtenidos por los diferentes grupos en la evaluación inicial de los talleres con niños, mujeres y hombres adultos muestran que, en los tres casos los sujetos tuvieron dificultad para definir los conceptos planteados. Es interesante que los niños fueran capaces de ejemplificar estos conceptos, mientras que con las mujeres y los hombres es evidente que están familiarizados con estos elementos. Estos resultados fueron comparados con una evaluación final, en la que es relevante que posterior a la experiencia de los talleres, los tres grupos niños, mujeres y hombres plasmar una definición más clara de los conceptos planteados, los niños describieron sus componentes; el grupo de mujeres logró mencionar ejemplos; y los tres grupos expresaron y reconocieron la importancia del medio natural en su comunidad.

Posteriormente, se indagó sobre el 
conocimiento que tienen los habitantes acerca de los problemas ambientales presentes y potenciales en su comunidad, y su capacidad para identificar la extensión de estos problemas, la importancia que le atribuyen, y los factores relacionados. Esto reflejado en la identificación de alteraciones del medio ambiente en sus diversas dimensiones y en su conocimiento de la magnitud de la crisis ambiental.

En esta segunda parte del cuestionario se apreció en la evaluación inicial, que la mayoría de ellos, no califica a los problemas mencionados como graves; sin embargo, las mujeres y hombre identificaron que en su comunidad existen algunos de los problemas planteados en los cuestionamientos como: contaminación del agua y el uso excesivo de agroquímicos .

En contraste, la evaluación final de los talleres con los tres grupos muestra, que se produjo un cambio importante en la forma de reflexión acerca de los posibles problemas en su comunidad. Los niños afirmaron que el uso de agroquímicos puede ser uno de los problemas más graves, esto coincidió con lo que mujeres y hombres manifestaron tanto en la evaluación inicial como final, pues expresaron que los problemas de contaminación por el uso de agroquímicos y la contaminación del agua, son los problemas más graves que se presentan en la comunidad.

Al cuestionar sobre la extensión que alcanzan los problemas ambientales; en la evaluación inicial la mayoría de los niños participantes opinan que sí existen problemas en su comunidad: el $33 \%$ de ellos opinaron que los problemas ambientales se producen en toda la comunidad, las mujeres señalaron que los problemas ambientales planteados pueden ser un peligro en el futuro, pero no actualmente; mientras que los hombres opinaron que los problemas ambientales solo se presentan en algunas áreas y son de poca importancia, no creen que tengan mucha relevancia. En contraste con la evaluación final, fue posible observar que más de la mitad los niños consideraron que los problemas ambientales sí afectan a toda la comunidad, las mujeres al igual que los hombres afirmaron que los problemas ambientales se producen principalmente en los campos agrícolas y están causando daños en la comunidad.

Posteriormente, se analizó la actitud ambiental de los habitantes de la comunidad y su decisión a incorporarse al cambio (PADILLA; LUNA, 2003); fue posible valorar la disposición real que tienen los habitantes de la zona para cuidar el medio ambiente y la apreciación que tienen los individuos sobre los agentes causantes de la contaminación ambiental, así como los actores sociales que deben intervenir para remediarla.

En la evaluación inicial, consideraron que es importante cuidar el medio ambiente; Además, reconocieron que no realizan prácticas de conservación y desconocen las acciones que deberían tomar, así como las normas relacionadas. También opinan que la escuela no influye lo suficiente en su formación ambiental y cuidado del entorno. Las mujeres y hombres consideran que estos tópicos son responsabilidad de la formación escolar y secundariamente de ellos mismos; sin embargo los niños indicaron que les compete principalmente a ellos.

En contraste, en la evaluación final muestra que niños, mujeres y hombres afirmaron que es importante cuidar el medio ambiente y están realizando acciones de conservación poniendo en práctica algunas acciones para cuidar su entorno. Los niños piensan que la escuela influye en su formación ambiental, pero hombres y mujeres adultos opinan que esa información debe estar complementada con acciones concretas. Posterior a los talleres expresan que el cuidado del entorno compete principalmente a ellos, y que es importante proponer alternativas y realizar acciones específicas en aspectos que están ocasionando problemas en su comunidad.

Por medio de la evaluación inicial(previa a los talleres) y final (posterior a la acción participativa), se observó un cambio sustancial en las respuestas de los participantes. Al principio algunos se mostraron apáticos al contestar, sin embargo al concluir los fue posible que los participantes lograran formar sus propios criterios, por medio del diálogo y la reflexión de los temas propuestos.

Según Balcázar (2003), el problema originado en la historia de explotación y alienación de los participantes, no les permite tomar la iniciativa para transformar su realidad. Es un papel crítico que el agente externo toma durante el proceso inicial; se 
trata de ayudar a los miembros de la comunidad o grupo, para que desarrollen una conciencia crítica de la realidad y realicen su potencial transformador. Esto coincide con lo expuesto por Freire (1970), quien afirma que los participantes pueden desarrollar la capacidad de descubrir su mundo con una óptica crítica, que les permita desarrollar habilidades de análisis, que pueden aplicar posteriormente a cualquier situación.

Otro aspecto importante, es la disposición de los individuos para realizar acciones que ayuden a cuidar su entorno, se mostraron comprometidos a trabajar a favor de sus recursos naturales, que consideraron importantes como medios de vida. Esto es de especial interés, ya que corrobora lo analizado por Balcázar (2003), quien plantea que los participantes en Investigación Acción Participativa, aprenden a entender su papel en el proceso de transformación de su realidad social, no como víctimas o como espectadores pasivos, sino como actores centrales en el proceso de cambio. Esto converge con lo propuesto por Freire (1970), quien argumenta que el individuo que adquiere una visión crítica del mundo, experimenta un cambio cualitativo que lo afecta y transforma por el resto de su vida.

En la fase de Acción-Reflexión-Acción, se evaluaron las actividades de los talleres. Previamente se eligieron aquellas que desarrollaran habilidades en las personas, además de considerar la disponibilidad del tiempo, se elaboró un cuadro (Tabla 02) en la cual se indican las actividades, objetivos, valores, aptitudes, actitudes y el número de participantes.

Tabla 02 - Ejemplo de tabla para evaluar las actividades realizadas en los talleres.

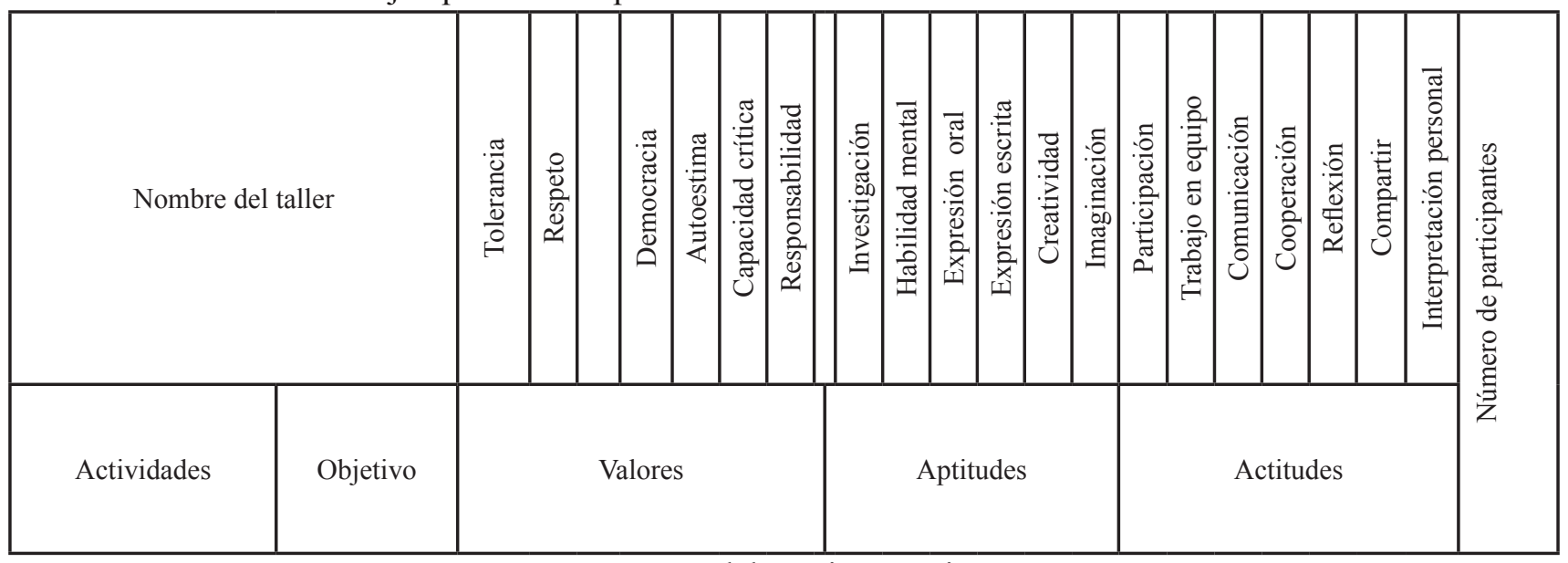

Fuente: Elaboración propia.

Se elaboraron cuadros para los 12 talleres con los diferentes grupos; se buscó que los participantes comprendieran y asumieran como principios de sus acciones y relaciones con los demás, valores tales como: tolerancia respeto, saber escuchar, democracia, autoestima, capacidad critica, responsabilidad.

La formación de estos valores sólo puede percibirse a través de las actitudes que los sujetos manifiestan en sus acciones, y en las opiniones que formulan espontáneamente, respecto a los hechos o situaciones que se presentan en su comunidad. Por esta razón, la educación ambiental a través de la participación, requiere un tratamiento vivencial, que fue considerado y tuvo sentido a lo largo de este estudio; ya que en cada una de las acciones y procesos que transcurrieron en los talleres, se vieron reflejados en acciones que muestran experiencias y nuevas formas de convivencia, cuyas bases sean el respeto a la dignidad humana, el diálogo, la tolerancia y el cumplimiento de los acuerdos entre individuos libres.

\section{Taller: Cuidado del Recurso Suelo}

El taller se desarrolló con los tres grupos, con un total de seis sesiones y participaron: 33 niños, 20 
mujeres y 10 hombres .

Los objetivos del taller fueron: valorar la importancia del suelo como recurso, desarrollar conocimientos y prácticas relacionados con el uso y conservación del suelo, elaborar la clasificación y separación de los residuos sólidos, implementar y practicar técnicas de composteo (GUTIÉRREZ et al., 2011 y 2012).

Para cumplir los objetivos de este taller se realizaron actividades como: analizar la importancia del suelo, discusiones con los participantes acerca del uso del suelo en su comunidad, identificar algunos métodos de conservación utilizados en Progreso Hidalgo, reflexionar sobre los posibles problemas que existen en su entorno, identificar posibles soluciones para estos problemas, y realizar acciones concretas para la solución de estos problemas.

Lo abordado en este taller, permitió a los participantes tomar conciencia de la importancia de la conservación de suelo, como uno de los recursos más importantes para la producción agrícola, ya que ellos dependen directamente de ella. Se observó que en el caso de hombres y mujeres, conocen algunas técnicas para la conservación de suelos, tales como: la rotación de cultivos, y la asociación de cultivos (maíz y fresa). En el caso de los niños se encontró que ellos están asociados con estas prácticas y las realizan, pero no tenían clara su utilidad.

En el ejercicio de reflexión acerca de los posibles problemas que pueden presentarse en el suelo de su comunidad, las mujeres, hombres y niños, expresaron que uno de los problemas más importantes, es el uso excesivo de productos agroquímicos que se aplican para aumentar la producción de fresa y otros cultivos florícolas. Otros problemas identificaron es que no se practica planeación para el uso del suelo; que los pobladores no saben qué hacer con sus residuos sólidos, por lo que algunas áreas de uso común están siendo contaminadas.

En este taller se realizaron actividades dinámicas para conocer y practicar la clasificación de los residuos sólidos, actividad en la que los niños presentaron un gran interés y realizaron una campaña en su comunidad para promover la clasificación de los residuos. Se ejemplificaron y practicaron técnicas de composteo; los participantes comprendieron que es una composta, para que es útil, los tipos de composta que existen y los tres grupos elaboraron abonos orgánicos con composta, utilizando residuos de sus hogares.

\section{Taller: Cuidado de los Recursos del Agua}

El taller se impartió en seis sesiones, son todos los grupos y participaron: 35 niños, 22 mujeres y 15 hombres adultos.

Los objetivos de estos talleres fueron: analizar la importancia del agua en la comunidad de Progreso Hidalgo y hacer una reflexión para generar alternativas y mejorar el uso del agua; aprender a trabajar en equipo para realizar acciones a favor de este recurso (GUTIÉRREZ et al., 2011 y 2012).

En este taller se realizaron actividades para entender el ciclo del agua, mediante el armado de un rompecabezas ellos explicaron la importancia de cada una de las fases del ciclo, y discutieron como el ser humano interviene en este proceso. Reflexionaron acerca de la importancia del agua en su comunidad y las posibles afectaciones a su entorno derivadas de la contaminación de este recurso o si este fuera insuficiente para cubrir sus necesidades.

Los participantes discutieron si en la comunidad utilizan algunas técnicas para captación y almacenamiento de agua, como manejan este recurso, si conocen y practican algún proceso para reciclar o reutilizar agua y si ellos consideran que se podría implementar algún método para reusar el agua, y como podría realizarse .

Los resultados alcanzados en este taller muestran que los participantes, comenzaron un proceso de toma de conciencia sobre la importancia del agua en su comunidad, la cual se ubica en una zona dedicada a la producción agrícola en la que este elemento es esencial para los cultivos. Ellos discutieron y analizaron acerca del potencial que poseen con el abundante recurso hídrico del que disponen, pues utilizan técnicas de almacenamiento de agua en bordos y distribución mediante canales de riego que se encuentran distribuidos por la comunidad. Ellos están organizados para la distribución de este importante recurso, y reconocieron que pueden 
mejorar el trabajo para desarrollar aspectos como: la participación activa de la comunidad, identificación de problemas relacionados con este recurso, elaborar propuestas para resolverlos, plantear proyectos relacionados con el cuidado del agua; así como realizar campañas para discutir la importancia de este recurso.

\section{Taller: Cuidado de la Vegetación}

El talleres se contó con la participación de 35 niños, 25 mujeres y 13 hombres adultos en promedio, seis sesiones por cada grupo.

Los objetivos de estos talleres fueron: Valorar la importancia que la vegetación tiene en Progreso Hidalgo, promover la participación de los sujetos para difundir la importancia de la vegetación en su comunidad, desarrollar experiencias sobre huertos familiares, hortalizas orgánicas, rotación de cultivos; y capacitar a los participantes para la instalación y práctica de técnicas para la conservación de la vegetación (GUTIÉRREZ et al., 2011 y 2012).

En este taller se llevaron a cabo actividades tales como: la discusión sobre la importancia de la vegetación en la comunidad de Progreso Hidalgo, la formación de equipos capaces de proponer como se puede difundir la importancia que tiene la vegetación en la comunidad. Se analizó la importancia y utilidad de un huerto familiar; la instalación y manejo de una hortaliza mediante la rotación de cultivos, distribución, selección, germinación y trasplante de plantas. Se destacaron los beneficios de un cultivo orgánico.

Los resultados de estos talleres fueron: la toma de conciencia sobre la importancia de la vegetación como recurso primordial para la existencia de la biodiversidad en la comunidad; la promoción práctica de su importancia a través de la conservación de los huertos familiares y la implementación de hortalizas de traspatio y la asociación de cultivos. Los tres grupos participaron activamente en el cultivo de hortalizas y mantenimiento de huertos familiares; el grupo de mujeres y hombres adultos hicieron la selección de las plantas que podían cultivarse en su comunidad según las condiciones que cada cultivo requiere.

La programación para la rotación de cultivos se elaboró basada en el conocimiento que los habitantes de la comunidad poseen sobre las condiciones edáficas y climáticas que se presentan según las estaciones del año. Realizaron la preparación de sustrato para la hortaliza, el grupo de niños se encargó de germinar las semillas para la hortaliza y del trasplante al lugar preparado para su plantación; también se encargaron de regar y vigilar que no se presentaran problemas de plagas en las hortalizas. El grupo de mujeres se encargó de promover la conservación de los huertos familiares existentes en la comunidad, de intercambiar especies, conservar y propagar las que están presentes en los huertos.

\section{Taller: Relaciones Humanas para vivir en Sociedad}

En este taller participaron 32 niños en 4 sesiones

El objetivo del taller fue: Promover el desarrollo de seres solidarios, preparados para vivir en armonía con otros, con mayores capacidades para su organización, trabajo en equipo y mejor convivencia.

Las actividades realizadas en este taller incluyeron la dinámica de las relaciones interpersonales en su comunidad, la valoración de la importancia de la confianza en las relaciones humanas, y la identificación de estrategias para mejor las relaciones.

La realización de este taller favoreció la motivación en los niños para expresar sus emociones, y tratar con respeto a las personas de su comunidad. Los niños fueron capaces de formular ejemplos de solidaridad entre las personas de su comunidad.

La experiencia del trabajo en equipo les permitió expresar cambios en su interacción social, y su disposición a ver el lado humano de las personas. Confirmaron su voluntad de poner en práctica lo aprendido, plantear soluciones prácticas a los problemas y actuar ante toda situación o problema; ellos expresaron su agrado por la experiencia. 


\section{Taller: Comunicación para aumentar la Comprensión}

El taller se realizó en cinco sesiones y participaron 22 mujeres.

El objetivo del taller fue desarrollar habilidades fundamentales en torno a la relación, comunicación, trabajo en equipo y compromiso. Las actividades realizadas en el taller incluyeron: la presentación personal ante el grupo; la expresión de sus expectativas respecto al taller, la reflexión sobre la comunicación y sus dificultades. Ellas vivieron la experiencia de transmitir información en variados mensajes y de reflexionar como se pierde, se distorsiona y se inventa; aprendieron a usar formas correctas de comunicación para resolver los conflictos de manera adecuada.

El taller permitió poder analizar los procesos y prácticas de comunicación comunes en la comunidad de Progreso Hidalgo. Esto les llevó a practicar herramientas de comunicación como interacción, intercambio, participación, creación, reflexión y acción. Identificaron los procesos de comunicación participativa, y los pusieron en práctica; se permitieron la expresión de otras voces más allá de las de los líderes. La comunicación comunitaria se aplicó como estrategia de comunicación popular, para analizar experiencias, identificar problemas y potencialidades; y de esta forma practicar poder y acción mediante la comunicación.

\section{Taller: Organización Comunitaria para el Desarrollo Local}

Este taller se realizo con la participación de 13 hombres, en cuatro sesiones.

El objetivo de este taller fue promover procesos participativos de organización, detectar obstáculos y fortalezas que actúan en las organizaciones comunitarias, generar el aprendizaje de nociones elementales de planificación y los conceptos necesarios para su organización.

Las actividades realizadas en este taller incluyeron: trabajo activo sobre participación en forma grupal, a partir de la reflexión sobre su contexto social y su realidad organizacional. Ellos estimularon la acción colectiva, mediante la identificación de problemas, análisis de las características de los problemas, y la propuesta de acciones prácticas.

El desarrollo de este taller permitió a los participantes adquirir herramientas para su organización, favoreció la mejora del trabajo comunitario, la toma de decisiones y participación comunitaria para el desarrollo sustentable. Se identificaron problemas y plantearon propuestas para resolverlos; propusieron proyectos sustentables, y plantearon escenarios deseados para su comunidad.

En este proyecto de investigación-acción, se consideró necesario tomar en cuenta la opinión de todos los participantes en los talleres; con el fin de evaluar el cumplimiento de los objetivos propuestos, replantear acciones, modificar procesos, e introducir nuevas variables.

Del análisis, interpretación y evaluación de los diferentes talleres, se encontró que el $98 \%$ de los participantes opinaron que los talleres les permitieron conocer nuevas técnicas de conservación y manejo ambiental sustentable; así como reafirmar algunas otras que ya conocían, y aclarar algunos conceptos que tenían sobre el ambiente. El 2\% de los participantes opina que los talleres fueron buenos y que deben contar con mayor difusión. El 86.3\% considera que sí cumplieron con las expectativas que ellos tenían y el $13,7 \%$ dijo que superaron sus expectativas.

El trabajo grupal de aprendizaje fue estimado por los participantes como novedoso, muy productivo, fácil y práctico; debido a que en la modalidad propuesta, se ha tenido en cuenta lo que cada participante piensa, sabe y dice. De este modo los participantes se involucraron y comprometieron de una manera más responsable con su comunidad, para participar en acciones tendientes a mejorar y conservar su entorno. Esto coincide con lo planteado por Chávez; Daza (2003), quienes afirman que el análisis debe ofrecer respuestas sobre las preguntas e hipótesis formuladas; esto determinará el grado de alcance de objetivos, la implicación y aprendizajes personales, el impacto en el ámbito de aplicación, y el manejo de los recursos utilizados.

Las conclusiones sobre los resultados de la valoración podrían ser inicio para nuevas líneas de trabajo. 


\section{CONSIDERACIONES FINALES}

La caracterización y el diagnóstico, proporcionaron el fundamento para la elaboración de los programas de estudio, así como para direccionar los talleres. La valoración de cada taller permitió comparar los resultados de la evaluación diagnóstica inicial y los de la evaluación final de los talleres por grupo; con el fin de determinar si el grado de conocimientos sobre el manejo sustentable de recursos naturales aumentó. En la evaluación inicial de niños, mujeres y hombres adultos fue posible observar que les faltaba precisión para definir el medio ambiente y los recursos naturales, aunque mencionaron ejemplos de estos temas. En la evaluación final se encontró que presentaron un cambio respecto a las repuestas dadas en la evaluación inicial; entonces fueron capaces de definir, ejemplificar y plantear alternativas para el cuidado sustentable de recursos naturales en su comunidad.

Los análisis realizados en este trabajo se basaron en situaciones que se presentan en la vida cotidiana de los pobladores de Progreso Hidalgo, tales como: las técnicas utilizadas para la producción de fresa, el planteamiento de problemas que pueden afectar a la comunidad y la solución de conflictos. Es posible afirmar que la participación de los sujetos en todas las actividades realizadas dentro y fuera de los talleres, fue decisiva y contribuyó a la formación de valores como tolerancia, respeto, democracia, capacidad crítica y responsabilidad.

Conforme se desarrollaron los talleres, los participantes fueron comprendiendo el significado y alcance de las actividades; y la importancia de la evaluación de las actividades. Esto les permitió aceptar con naturalidad, las apreciaciones que hacían los demás participantes; así asumieron con mejor disposición contestar el cuestionario de evaluación final; y comprendieron la importancia de detectar sus debilidades y reconocer sus fortalezas, y de esta manera proponer acciones para mejorar su entorno.

Experiencias como ésta permiten acercarse al Proceso Educativo de Investigación Acción, como aproximaciones al trabajo participativo, como plantea Balcázar (2003), los problemas psicosociales no van a desaparecer sin intervenciones directas, $\mathrm{y}$ los gobiernos no tienen suficientes recursos como para darse el lujo de excluir a los usuarios del proceso de cambio. La gente misma tiene que involucrarse, pues su pasividad no genera soluciones.

La educación popular ambiental incorpora los planteamientos ecológicos de la educación ambiental y los aspectos sociopolíticos de la educación popular (REYES, 1994); esta definió la elaboración de los talleres en la comunidad de Progreso Hidalgo, al retomar presupuestos como son: la reflexión de lo particular a lo general, partir de la práctica a la teoría y después renovarla, la valorización y revalorización del saber popular, una asunción amplia del proceso educativo, siempre con la participación de los pobladores de esta comunidad rural.

La educación popular ofrece los elementos conceptuales y prácticos que pueden ayudar a adquirir una visión sistemática de un entorno natural y social, a través de la acumulación de experiencias de campo con los participantes, que permiten desarrollar un conocimiento más completo de la efectividad de las acciones realizadas en ciertas condiciones. Lo más importante de este ejercicio es el diálogo y la reflexión que llevan al desarrollo de conciencia crítica en los participantes, de acuerdo con Freire (1970). La participación en cualquier ámbito permite desarrollar un sentido de pertenencia y aumenta el grado de compromisos de los colaboradores.

La aplicación de la metodología de la investigación acción participativa incluye supuestos importantes para el desarrollo de experiencias vinculadas al desarrollo rural sustentable que pueden ser aplicadas en otras comunidades, pues al igual que en esta experiencia, las demás deben coincidir en cuanto al papel protagónico y participativo de la población local, la comunidad; y el espacio como escenario para realizar este tipo de prácticas.

Asimismo, en el desarrollo de este tipo de experiencias debe existir como punto de partida una visión holística del ambiente, que incluye los aspectos naturales, físicos, sociales y culturales además de una identificación de los sujetos ambientales, donde las personas son protagonistas de los problemas y soluciones de sus propias comunidades. 


\section{REFERENCIAS}

BALCAZAR, F. E. Investigación acción participativa (IAP): Aspectos conceptuales y dificultades de implementación. Fundamentos en Humanidades, San Luis, ano IV, n. I/ II, 2003, pp. 59-77. Disponible en: $<$ http://www.redalyc.org/articulo.oa?id=18400804>. Fecha de consulta: 24 jan. 2014.

CÁRDENAS, J. L. Definición de un marco teórico para comprender el concepto del desarrollo sustentable. Revista INVI, Norteamérica, Santiago, v. 13, n. 33, 1998, pp. 03-20. Disponible en: <http://revistainvi. uchile.cl/index.php/INVI/article/view/228/990>. Fecha de consulta: 25 jan. 2014.

CHÁveZ, M. G.; DAZA, S. J. C. Reflexión metodológica sobre la aplicación concreta de la Investigación Acción Participativa (IAP) en contextos rurales del estado de Colima. Estudios sobre las Culturas Contemporáneas, Colima, v. IX, n. 17, 2003, pp. 115-146. Disponible en: < http://www.redalyc. org/articulo.oa? $\mathrm{id}=31601707>$. Fecha de consulta: 24 jan. 2014.

FALS, B. O.; RODRÍGUEZ, B. C. Investigación Participativa. Montevideo: La Banda Oriental. 1987.

FREIRE, P. Pedagogía del oprimido. Montevideo: Tierra Nueva, 1970.

GHISO, A. Acercamientos: el taller en procesos de investigación interactivos. Estudios sobre las Culturas Contemporáneas, Colima, v. V, n. 9, 1999, pp. 141153. v.141-153. Disponible en: $<$ http://www.redalyc. org/articulo.oa? id=31600907>. Fecha de consulta: 24 jan. 2014.

GOBIERNO DEL ESTADO DE MÉXICO. Atlas del Estado de México. 1995.

GOBIERNO DEL ESTADO DE MÉXICO. Dirección General de Protección Civil. Atlas de Riesgos del Estado de México. 2a. versión. 2000.

GUtiÉRReZ, C. J. G.; AGUilerA, G. L. I.;
GONZÁLEZ, E. C. E.; JUAN, P.J.I. Evaluación preliminar de la sustentabilidad de una propuesta agroecológica, en el Subtrópico del Altiplano Central de México. Tropical and Subtropical Agroecosystems, Mérida, v. 14, n. 2, 2011, pp. 567-580.

GUTIÉRREZ, C. J. G.; AGUILERA, G. L. I.; GONZÁLEZ, E. C. E.; JUAN, P. J. I. Evaluación de la sustentabilidad posterior a una intervención agroecológica, en el Subtrópico del Altiplano Central de México. Tropical and Subtropical Agroecosystems, Mérida, v. 15, n. 1, 2012, pp. 15-24.

INSTITUTO NACIONAL DE ESTADÍSTICA, GEOGRAFÍA E INFORMÁTICA. Carta de uso del suelo. Escala 1:50000, Clave E14 A58. México. 1982.

INSTITUTO NACIONAL DE ESTADÍSTICA Y GEOGRAFÍA (INEGI). Censo de Población y Vivienda, 2010.

JUAN, P. J. I. Tiempo con dinero y tiempo sin dinero. Agricultura tradicional y comercial en una zona de transición ecológica del Estado de México. (Tesis doctoral). Universidad Iberoamericana, México, 2003.

JUAN, P. J. I. Manejo del ambiente y riesgos ambientales en la región fresera del Estado de México, 2006.

JUAN, P. J. I. Agricultura tradicional y comercial en una zona de transición ecológica de México. Argentina: Dunken, 2007.

JUAN, P. J. I.; GUTIÉRREZ C. J. G.; BALDERAS P. M. Á.; ANTONIO N. X. La mujer campesina y el manejo de huertos. Una estrategia para la alimentación de las familias mexicanas. LEISA Revista agroecología, Lima, v. 25, n. 3, 2009, pp. 31-33.

PADILLA Y SOTELO, L. S.; LUNA A. M. Percepción y conocimiento ambiental en la costa de Quintana Roo: una caracterización a través de encuestas: Investigaciones Geográficas. Boletín UNAM, México, n. 52, 2003, pp. 99-116. DOI: http:// dx.doi.org/10.14350/rig.30335 
REYES, J. La Educación Popular y la Dimensión Ambiental del Desarrollo. Documento de Discusión. Asamblea de CEAAL, Santiago de Chile, 1994.

SAUVÉ, L. La educación ambiental y la globalización: desafíos curriculares y pedagógicos. Revista Iberoamericana de Educación, Madrid, n. 41, 2006, pp. 83-101.

SELENER, D. Participatory action research and social change. NY: Cornell University Participatory Action Research Network, 1997.

SHANIN, T. Naturaleza y Lógica de la Economía Campesina. Barcelona: Anagrama, 1976.

TURRENT, FERNÁNDEZ, A.; CORTÉS, FLORES, J L. Ciencia y tecnología en la agricultura mexicana: I. Producción y sostenibilidad. Terra Latinoamericana, Chapingo, v. 23, n. 2, 2005, pp. 265-272. Disponible en: $<$ http://www.redalyc.org/articulo.oa?id=57323214>. Fecha de consulta: 24 jan. 2014. 